\title{
Les vacances d'été des élèves des écoles primaires de quelques localités vaudoises (1963)
}

\author{
Laurent Bridel
}

\section{Introduction}

Dans la géographie du tourisme et des vacances, deux points de vue principaux se complètent et permettent de mieux faire ressortir les nuances de la réalité. Il s'agit d'une part de l'étude des flux de touristes, de leur hébergement et de leurs activités dans une région touristique et d'autre part de l'analyse des vacances et des buts de voyage de la population indigène. A l'échelle d'un grand pays où l'immense majorité des touristes sont des nationaux, les deux points de vue tendent à se confondre (par exemple pour l'ensemble de la Grande-Bretagne ou de la République fédérale allemande). Il n'en est absolument pas de même à l'échelle réduite d'un canton suisse, d'autant plus que les vacanciers étrangers forment un contingent considérable.

Les documents qui sont analysés ici sont un apport précieux à notre connaissance de la géographie sociale suisse, car jusqu'ici ce n'est guère que le premier point de vue, l'étude du tourisme dans une région, qui a prévalu en Suisse.

\section{a) Origine des documents étudiés}

Les chiffres dont nous disposons ont été récoltés à des fins tout à fait pratiques. Au cours de ces dernières années, la commission scolaire de Lausanne avait souvent dû prendre en considération des réclamations de parents qui avaient loué un chalet pour un mois de vacances en été et qui perdaient le bénéfice d'au moins une semaine de location, soit en juillet, soit en août, du fait des dates des vacances, à cheval sur ces deux mois. Afin d'évaluer plus précisément le nombre d'élèves touchés par ce découpage malencontreux, la commission scolaire demanda à tous les enseignants des classes primaires (classes enfantines, semi-enfantines, primaires, primaires supérieures, ménagères, d'orientation, spéciales) d'effectuer une enquête auprès de leurs élèves, pour connaître leur utilisation du temps pendant les vacances d'été 19631

Les résultats, collationnés en octobre 1963, ont été envoyés à plusieurs communes du canton, en les priant de bien vouloir procéder à la même enquête. Une dizaine de villes ou de bourgs répondirent favorablement à cette demande, ce qui permet de comparer les données de Lausanne avec celles d'autres parties du canton.

\section{b) S'agit-il de géographie sociale?}

J'ai parlé à la fois de géographie du tourisme et des vacances et de géographie sociale. Ces deux concepts doivent être distingués. La géographie des vacances des enfants des écoles vaudoises consisterait au premier chef à connaître les lieux où ces élèves se sont rendus, éventuellement leur durée de séjour, et cette analyse permettrait d'établir des corrélations spatiales entre le lieu de domicile et ses caractéristiques d'une part (altitude, climat, grandeur de la localité, etc.) et les caractéristiques dú lieu de vacances. Malheureusement, le questionnaire est beaucoup trop vague; il permet tout au plus de distinguer ceux qui sont restés sur place, ceux qui sont allés en un lieu de vacances suisse et ceux qui sont partis à l'étranger.

C'est pourquoi, il s'agit en fait de renseignements de géographie sociale. Les réponses aux questions sont avant tout des renseignements sociologiques. C'est grâce au fait qu'ils sont fournis localité par localité et quartier par quartier pour Lausanne que l'on peut les utiliser spatialement. La comparaison de faits sociologiques dans l'espace sera de l'écologie pour le sociologue et de la géographie sociale pour le géographe. Les quelques pages qui y suivent sont donc un apport à la géographie sociale du canton de Vaud en 1963.

\section{Critique des documents}

Etant donné que nous cherchons à établir une relation entre les caractéristiques sociales de la population et son mode de vacances, chacune des questions de la formule de la Commission scolaire doit être examinée de ce point de vue.

Tout d'abord, nous ne sommes informés que sur les vacances des enfants en âge de scolarité obligatoire et encore à l'exclusion des écoles secondaires.

Le camping nous apparaît comme un mode d'hébergement moins coûteux que l'hôtel ou le chalet et, par conséquent, mieux à la portée des catégories peu aisées de la population. Cependant, il nécessite presque obligatoirement l'usage de l'auto et il est aussi apprécié par des personnes du toutes catégories, ce qui en fait un indicateur quelque peu ambivalent.

En revanche, le fait de rester à domicile pendant 
Tableau 1

Genre de classes touchées par l'enquête

\begin{tabular}{|c|c|c|c|c|c|c|c|c|c|}
\hline Localités & 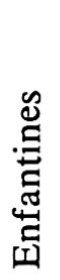 & 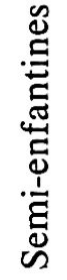 & 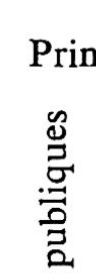 & 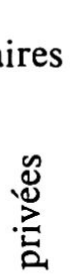 & 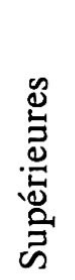 & 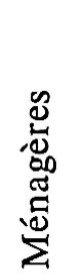 & $\begin{array}{l}\stackrel{N}{0} \\
\dot{0} \\
\dot{0}\end{array}$ & 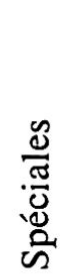 & 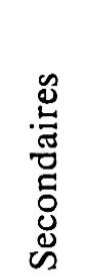 \\
\hline Lausanne & $\mathrm{x}$ & $\mathrm{x}$ & $\mathrm{x}$ & & $\mathrm{x}$ & $\mathbf{x}$ & $\mathrm{x}$ & $\mathrm{x}$ & \\
\hline Pully & & & $\mathrm{x}$ & & $\mathrm{x}$ & $\mathbf{x}$ & $\mathbf{x}$ & & \\
\hline Nyon & $\mathbf{x}$ & $\mathrm{x}$ & $\mathrm{x}$ & $\mathrm{x}$ & $\mathbf{x}$ & & & & $(x)^{3}$ \\
\hline Rolle & $\mathrm{x}$ & $\mathrm{x}$ & $\mathrm{x}$ & & & $\mathrm{x}$ & & & \\
\hline $\begin{array}{l}\text { Montreux } \\
\text { La Tour-de-Peilz }\end{array}$ & $\mathrm{x}$ & $\mathrm{x}$ & $\begin{array}{c}\mathbf{X} \\
\text { prime }\end{array}$ & & $\mathrm{x}$ & & & & \\
\hline $\begin{array}{l}\text { Payerne } \\
\text { Yverdon }\end{array}$ & $\mathrm{x}$ & $\mathrm{x}$ & $\begin{array}{c}\mathrm{x} \\
\text { prima }\end{array}$ & & & & & & $\mathrm{x}$ \\
\hline $\begin{array}{l}\text { Grandson } \\
\text { Vallorbe }\end{array}$ & $\mathrm{x}$ & $\mathrm{x}$ & $\begin{array}{c}\mathbf{X} \\
\text { prima }\end{array}$ & & $\mathrm{x}$ & $\mathrm{x}$ & & & $\mathbf{X}$ \\
\hline
\end{tabular}

toutes les vacances paraît généralement lié à de faibles moyens matériels (questions 6 et 8 ).

Le fait d'aller à l'étranger peut généralement être interprété dans le sens contraire.

Le questionnaire n'étant destiné qu'à éclairer un organisme sur un problème bien particulier, il comporte des faiblesses lorsque l'on cherche à l'analyser dans d'autres buts:

- certaines questions sont trop vagues;

- d'autres sont trop restrictives. Pourquoi, à la quatrième, ne parler du camping qu'en famille?

- d'autres auraient mérité d'être subdivisées (par exemple, la septième qui mêle colonies de vacances et séjours chez des parents);

- certains types vacances échappent à l'enquête: qu'en est-il du camp d'éclaireurs par exemple?

- selon la localité ou l'enseignant, il y a eu des différences d'interprétation.

C'est pourquoi, notre analyse ne peut être considérée que comme une hypothèse de recherches sur la base d'un matériel difficile à évaluer.

\section{Comparaison entre les localités}

Bien qu'ayant touché la plupart des localités importantes du canton, il n'est possible de considérer les réponses des 12784 élèves comme représentatives de l'ensemble des villes du canton. Tout d'abord des localités de l'importance de Vevey, Morges, Orbe, Le Chenit, Moudon et Ste-Croix manquent; d'autre part, il ne s'est pas agi d'un sondage préparé selon des règles scientifiques. Par conséquent, nos con- sidérations ne concerneront que les localités touchées par l'enquête.

Dans trois communes, les élèves des collèges ont aussi été interrogés. Malheureusement, ce n'est qu'à Rolle que l'on a pu les déduire du total. Il semble que les résultats de Payerne et de Vallorbe en ont été un peu déviés.

\section{Tableau 2}

\section{Nombre d'élèves interrogés}

Localités

Nombre

Lausanne

Pully

Nyon

Rolle

(non compris 118 collégiens secondaires)

Montreux

La Tour-de-Peilz

Payerne

Yverdon

Grandson

Vallorbe

455

Total

12784

Il faut ajouter que, dans certaines localités, les élèves des classes primaires supérieures ou ménagères viennent en partie de communes voisines. Notre étude ne comporte donc pas que des réponses de citadins, mais cela ne représente qu'un faible pourcentage de l'ensemble. 


\begin{tabular}{|c|c|c|c|c|c|c|c|c|c|c|c|c|}
\hline Localités & 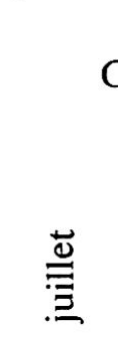 & 莽 & 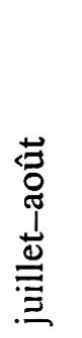 & Total & U్రు & 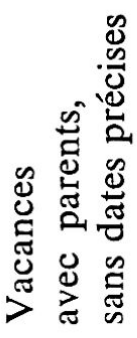 & 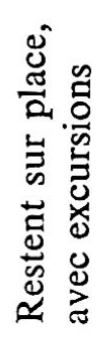 & 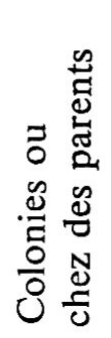 & 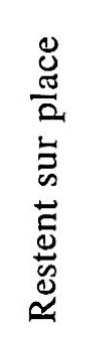 & Total & 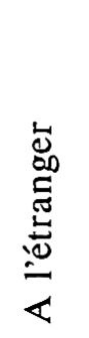 & 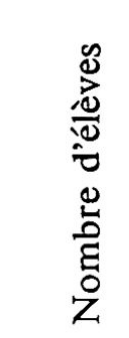 \\
\hline No de la question & 1 & 2 & 3 & $1-3$ & 4 & 5 & 6 & 7 & 8 & 6 et 8 & 9 & \\
\hline Lausanne & 4 & 5 & 3 & 12 & 16 & 24 & 12 & 43 & 7 & 19 & 26 & 5331 \\
\hline Pully & 5,4 & 8,2 & 3,2 & 17 & 14 & 25,4 & 14,4 & 33 & 7,6 & 22 & 38 & 501 \\
\hline Nyon & 2,8 & 3,7 & 2 & 8,5 & 7,9 & 18,2 & 14,8 & 36,8 & 17,6 & 32,4 & 21,3 & 1000 \\
\hline Rolle & P 2 & 8 & 0,8 & 11 & 6 & 13,4 & 14 & 26,5 & 16 & 30 & 13,4 & 238 \\
\hline & S 0,8 & 8,5 & 0,8 & 10 & 15,3 & 14,4 & 16,9 & 25,5 & 11,9 & 28,8 & 5,9 & \\
\hline Montreux & 3,3 & 4,8 & 1,9 & 10 & 10,7 & 23,9 & 18 & 46,9 & 18,2 & 36,2 & 20,9 & 1205 \\
\hline La Tour-de-Peilz & 2,7 & 6,3 & 1,7 & 10,7 & 12 & 30 & 10 & 35 & 10 & 20 & 26,5 & 600 \\
\hline Payerne & 2,3 & 2,5 & 1,5 & 6,3 & 11 & 17,4 & 14 & 39,5 & 23,2 & 37,2 & 14,0 & 1069 \\
\hline Yverdon & 1 & 1,9 & 0,6 & 3,5 & 6,1 & 14,7 & 10,7 & 25,5 & 10,9 & 21,6 & 11,5 & 2101 \\
\hline Grandson & 0,4 & 0,8 & 0 & 1,2 & 7,7 & 9,1 & 11,1 & 25,4 & 30 & 41,1 & 15,5 & 284 \\
\hline Vallorbe & 0,7 & 1,8 & 0 & 2,5 & 10 & 32 & 19 & 41 & 15 & 34 & 24 & 455 \\
\hline Valeurs extrêmes & & & & & & & & & & & & 12784 \\
\hline Minimum & 0,4 & 0,8 & 0,6 & 1,2 & 5 & 9,1 & 10 & 25,4 & 7 & 19 & 11,5 & \\
\hline Maximum & 5,4 & $\begin{array}{c}\mathbf{8 , 5} \\
\text { ou } \\
8,2\end{array}$ & 3,2 & 17 & 16 & 30 & 19 & 46,9 & 30 & 41,4 & 38 & \\
\hline
\end{tabular}

\section{a) Principaux résultats généraux (voir tableau 3)}

Les enfants qui ont passé tout ou partie des vacances avec leur famille dans un chalet ou un appartement de vacances ne sont qu'en nombre limité puisque le plus fort pourcentage (Pully) n'est que de 17. En général, il oscille autour de 10 à $12 \%$ pour les autres villes. Bien que, dans les stations suisses, la majeure partie des utilisateurs de chalets soient des Suisses, on voit que, par rapport à la population totale, ce n'est qu'une faible proportion. On sait par ailleurs ${ }^{4}$ que les ménages avec enfants sont particulièrement nombreux parmi les touristes en chalets; notre analyse nous rappelle ainsi que la majeure partie des enfants touristes échappe à tout dénombrement officiel direct, si l'on excepte l'examen du produit des taxes de séjour.

La proportion des enfants ayant campé oscille entre 6 et $16 \%$ (Rolle et Lausanne). Par conséquent, les campeurs, chez les enfants, sont aussi nombreux que ceux passant les vacances en chalet. C'est une constatation intéressante qui montre l'importance de ce type de vacances pour les familles.

La question 5, trop difficile à juger, est laissée de côté. Faute de mieux, nous avons regroupé les résultats des questions 6 et 8 , ce qui nous amène à dire que 20 à $40 \%$ des enfants ne changent pas de domicile pendant leurs vacances. Cela peut vouloir dire bien des choses: a) La santé ou le caractère de l'enfant ne permettent pas un changement de milieu, même temporaire. On voit qu'à Lausanne, les classes spéciales sont celles où le pourcentage est le plus fort à ces deux questions.

b) Les parents n'ont pas les moyens financiers d'envoyer leurs enfants en vacances ailleurs.

c) Les parents n'ont pas les moyens financiers de passer les vacances familiales ailleurs.

Rappelons que l'Institut suisse d'opinion publique (ISOP) a trouvé que, en 1961, 53\% des Suisses s'absentaient pendant leurs vacances contre $62 \%$ en $1965^{5}$. Ces chiffres concordent très bien avec l'éventail des résultats obtenus pour les localités vaudoises examinées.

La question 7 mélange - nous l'avons vu - deux aspects différents l'un de l'autre: les séjours chez des parents et la participation à une colonie de vacances. Ces deux modes de vacances sont parmi les moins coûteux, mais ils n'ont pàs les mêmes implications sociales. En outre, le déplacement vers des parents peut être à plus longue distance que lors d'un départ en colonie de vacances.

Pour tenter de savoir quelles sont les localités où les colonies occupent une place plus importante par rapport aux séjours chez des parents, il est possible de recenser le nombre de places dans les colonies 
dépendant de chaque commune. Encore faut-il ajouter qu'il existe plusieurs institutions de vacances qui ne sont pas liées à une commune en particulier, ce qui modifie l'image ci-dessous.

\section{Tableau 4}

Réponses positives à la question 7 et places dans les colonies de vacances

\begin{tabular}{|c|c|c|}
\hline Localités & 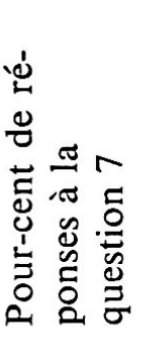 & 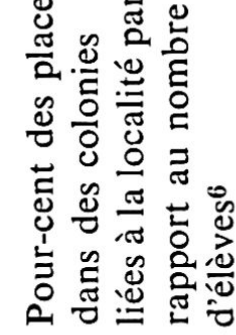 \\
\hline 1 Grandson & 25,4 & 12,7 \\
\hline 2 Yverdon & 25,5 & 2,5 \\
\hline 3 Rolle & 26,5 & 0 \\
\hline 4 Pully & 33 & 12 \\
\hline 5 La Tour-de-Peilz & 35 & 5 \\
\hline 6 Nyon & 36,8 & 8,7 \\
\hline 7 Payerne & 39,5 & 8,4 \\
\hline 8 Vallorbe & 41 & 6,6 \\
\hline 9 Lausanne & 43 & 9,4 \\
\hline 10 Montreux & 46,9 & 9,2 \\
\hline
\end{tabular}

Le nombre de places dans les colonies de vacances dépendant de la commune n'est pas proportionnel aux réponses à la question 7 . Est-ce dû au nombre variable suivant le lieu d'élèves allant chez des parents? On pourrait, à titre d'hypothèse, dire que chaque place dans une colonie est occupée en moyenne par deux élèves au cours de l'été. Cette estimation s'appuie sur le fait qu'à Montreux, seule commune où l'indication a été fournie, $17,4 \%$ des élèves ont été en colonie, alors que le nombre de places correspond à $9,2 \%$ du nombre total d'écoliers. Si l'on suivait cette idée, on obtiendrait l'image suivante:

\section{Tableau 5}

Pour-cent d'enfants en colonies et pour-cent séjournant chez des parents

$\begin{array}{lll}\text { Localités } & \begin{array}{l}\text { Pour-cent d'élèves } \\ \text { en colonies }\end{array} & \begin{array}{l}\text { Pour-cent d'élèves } \\ \text { ayant séjourné } \\ \text { chez des proches }\end{array}\end{array}$

$\begin{array}{lcc}\text { Grandson } & 25,4 & 0 \\ \text { Pully } & 24 & 9 \\ \text { Nyon } & 17,5 & 19,3 \\ \text { Yverdon } & 5 & 20,5 \\ \text { Payerne } & 17 & 22,5 \\ \text { Lausanne } & 19 & 24 \\ \text { La Tour-de-Peilz } & 10 & 25 \\ \text { Rolle } & 0 & 26,5 \\ \text { Vallorbe } & 13 & 28 \\ \text { Montreux } & 17,47 & 29,47\end{array}$

Pour Pully et Grandson, ces chiffres ne paraissent pas convenir. Toute notre connaissance du caractère aisé de Pully nous porte à croire que le nombre d'élèves en colonies de vacances doit être un des plus réduits. L'explication viendrait sans doute du fait que la commune loue ou prête ses installations à d'autres groupes, ce qui permettrait de changer le chiffre. On peut imaginer un phénomène assez semblable dans le cas de Grandson.

Ceci mis à part, l'image donnée serait assez satisfaisante si l'on part de la supposition que la forme de vacances chez des proches est répandue, de manière relativement indépendante du niveau de revenu, car le déplacement peut se faire à des distances et selon des modalités très variées. Dans l'ensemble environ un quart des élèves vont séjourner en vacances chez des parents.

Enfin, la question 9 indiquant le nombre d'élèves qui se sont rendus à l'étranger donne des valeurs particulièrement divergeantes les unes des autres, allant de $11,5 \%$ à Yverdon à 38\% à Pully, ce qui révèle aussi un degré d'évolution variable à la fois dans les moyens matériels et dans la mentalité.

En admettant que les réponses aux questions 6 et 8 s'additionnent, on peut dresser le tableau suivant de la répartition spatiale des lieux de vacances.

\section{Tableau 6}

\section{Répartition spatiale des lieux de vacances}

Pour-cent d'élèves ayant passé leurs vacances

\begin{tabular}{|c|c|c|c|}
\hline Localités & 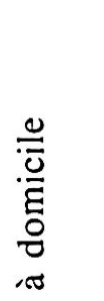 & 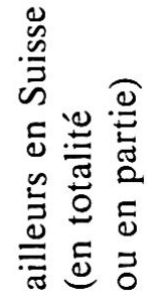 & 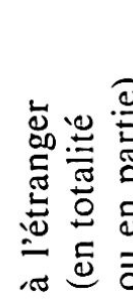 \\
\hline Lausanne & 19 & 55 & 26 \\
\hline La Tour-de-Peilz & 20 & 53,5 & 26,5 \\
\hline Yverdon & 21,6 & 66,9 & 11,5 \\
\hline Pully & 22 & 40 & 38 \\
\hline Rolle & 30 & 56,6 & 13,4 \\
\hline Nyon & 32,4 & 46,3 & 21,3 \\
\hline Vallorbe & 34 & 42 & 24 \\
\hline Montreux & 36,2 & 42,9 & 20,9 \\
\hline Payerne & 37,2 & 48,8 & 14 \\
\hline Grandson & 41,1 & 43,4 & 15,5 \\
\hline
\end{tabular}

Alors que la première colonne montre l'importance des familles peu mobiles pendant les vacances et que la dernière indique la part des familles ayant les moyens et le désir de se dépayser hors de nos frontières, la catégorie centrale, résultante des deux autres est plus pauvre en enseignements. Le pourcent important d'élèves de Vallorbe allant à l'étran- 
Tableau 7 Localités par ordre de pour-cent décroissant (D-E) ou croissant (A-C)

\begin{tabular}{|c|c|c|c|c|c|c|c|c|c|}
\hline $\begin{array}{l}\text { Pour-cent } \\
\text { en chalets } \\
\text { A }\end{array}$ & & $\begin{array}{l}\text { Pour-cent } \\
\text { de campeurs } \\
\text { B }\end{array}$ & & $\begin{array}{l}\text { Pour-cent } \\
\text { à l'étranger } \\
\text { C }\end{array}$ & & $\begin{array}{l}\text { Pour-cent } \\
\text { en colonies o } \\
\text { chez des pare } \\
\text { D }\end{array}$ & & $\begin{array}{l}\text { Pour-cent } \\
\text { de vacances } \\
\text { à domicile } \\
\text { E }\end{array}$ & \\
\hline 1. Pully & 17 & 1. Lausanne & 16 & 1. Pully & 38 & 1. Grandson & 25,4 & 1. Lausanne & 19 \\
\hline 2. Lausanne & 12 & 2. Pully & 14 & 2. La Tour & 26,5 & 2. Yverdon & 25,5 & 2. La Tour & 20 \\
\hline 3. Rolle & 10,8 & 3. La Tour & 12 & 3. Lausanne & 26 & 3. Rolle & 26,5 & 3. Yverdon & 21,6 \\
\hline 4. La Tour & 10,7 & 4. Payerne & 11 & 4. Vallorbe & 24 & 4. Pully & 33 & 4. Pully & 22 \\
\hline 5. Montreux & 10 & 5. Montreux & 10,7 & 5. Nyon & 21,3 & 5.La Tour & 35 & 5. Rolle & 30 \\
\hline 6. Nyon & 8,5 & 6. Vallorbe & 10 & 6. Montreux & 20,9 & 6. Nyon & 36,8 & 6. Nyon & 32,4 \\
\hline 7. Payerne & 6,3 & 7. Nyon & 7,9 & 7. Grandson & 15,5 & 7. Payerne & 39,5 & 7. Vallorbe & 34 \\
\hline 8. Yverdon & 3,5 & 8. Grandson & 7,7 & 8. Payerne & 14 & 8. Vallorbe & 41 & 8. Montreux & 36,2 \\
\hline 9. Vallorbe & 2,5 & 9. Yverdon & 6,1 & 9. Rolle & 13,4 & 9. Lausanne & 43 & 9. Payerne & 37,2 \\
\hline 10. Grandson & 1,2 & 10. Rolle & 6 & 10. Yverdon & 11,5 & 10. Montreux & 46,9 & 10. Grandson & 41,1 \\
\hline
\end{tabular}

ger frappe pour une aussi petite localité. Il est bien entendu lié à la situation frontalière du bourg.

\section{b) Essai de classement}

Afin de pousser un peu plus loin notre recherche, nous allons chercher à classer les localités analysées en quelques catégories afin de mieux distinguer si les résultats de l'enquête peuvent être mis en parallèle avec d'autres données.

Nous admettons que le pour-cent de réponses pour les vacances en chalets et le pour-cent d'enfants ayant été à l'étranger vont dans le même sens, alors que le pour-cent de ceux qui sont restés à domicile est de sens contraire. Les premiers seraient des indicateurs de milieux aisés alors que le dernier serait le signe d'une catégorie sociale plus modeste. A titre de comparaison, nous prendrons en considération également le pour-cent de campeurs et les résultats de la question 7.

Les éléments servant à ce classement sont donnés au tableau 7 où les localités sont indiquées par ordre croissant pour les trois premières caractéristiques et par ordre décroissant pour les deux dernières.

En donnant la note 10 à la localité de $1^{\text {er }}$ rang, puis 9 à celle de second rang et ainsi de suite, nous obtenons un classement en cinq groupes, avec un maximum de 50 points (voir carte 2 ).

$$
\begin{array}{lr}
\text { Pully } & 43 \\
\text { Lausanne, La Tour-de-Peilz } & 39 \\
\text { Nyon, Rolle } & 25 \\
\text { Yverdon, Montreux, Vallorbe } & 21-23 \\
\text { Grandson, Payerne } & 19-20
\end{array}
$$

Ces groupes sont indiqués dans l'ordre décroissant de mobilité et d'apparence sociale aisée. Nous allons maintenant comparer cette classification avec quelques caractéristiques démographiques. c) Comparaison des résultats avec quelques données démographiques

\section{Tableau 8}

Nombre d'habitants des localités en 1963

Localités de plus de

100000 habitants

\section{Lausanne}

Localités de 10000 à

Yverdon, Montreux, 20000 habitants Pully, La Tour-de-Peilz

Localités de 5000 à

10000 habitants

Nyon, Payerne

Localités de moins de 5000 habitants

Vallorbe, Grandson, Rolle

Il ressort de ce premier tableau que notre classement n'est pas fonction du nombre d'habitants. Il n'en est pas de même avec les grandes catégories d'activité économique.

\section{Tableau 9}

Pour-cent de personnes occupées dans le grand groupe d'activité tertiaire

(1960, recensement fédéral)
Plus de $65 \% \quad$ Pully
55 à $64,9 \%$ Lausanne, Montreux,
La Tour-de-Peilz
45 à $54,9 \%$
Rolle, Payerne, Nyon
35 à $44,9 \%$
Grandson, Yverdon, Vallorbe

Il est évident que le tableau aurait aussi pu être établi en fonction de la part de population active dans l'industrie et l'artisanat, car l'agriculture ne représente une part notable qu'à Grandson $(11,2 \%)$ et à Payerne $(9,9 \%)$. 


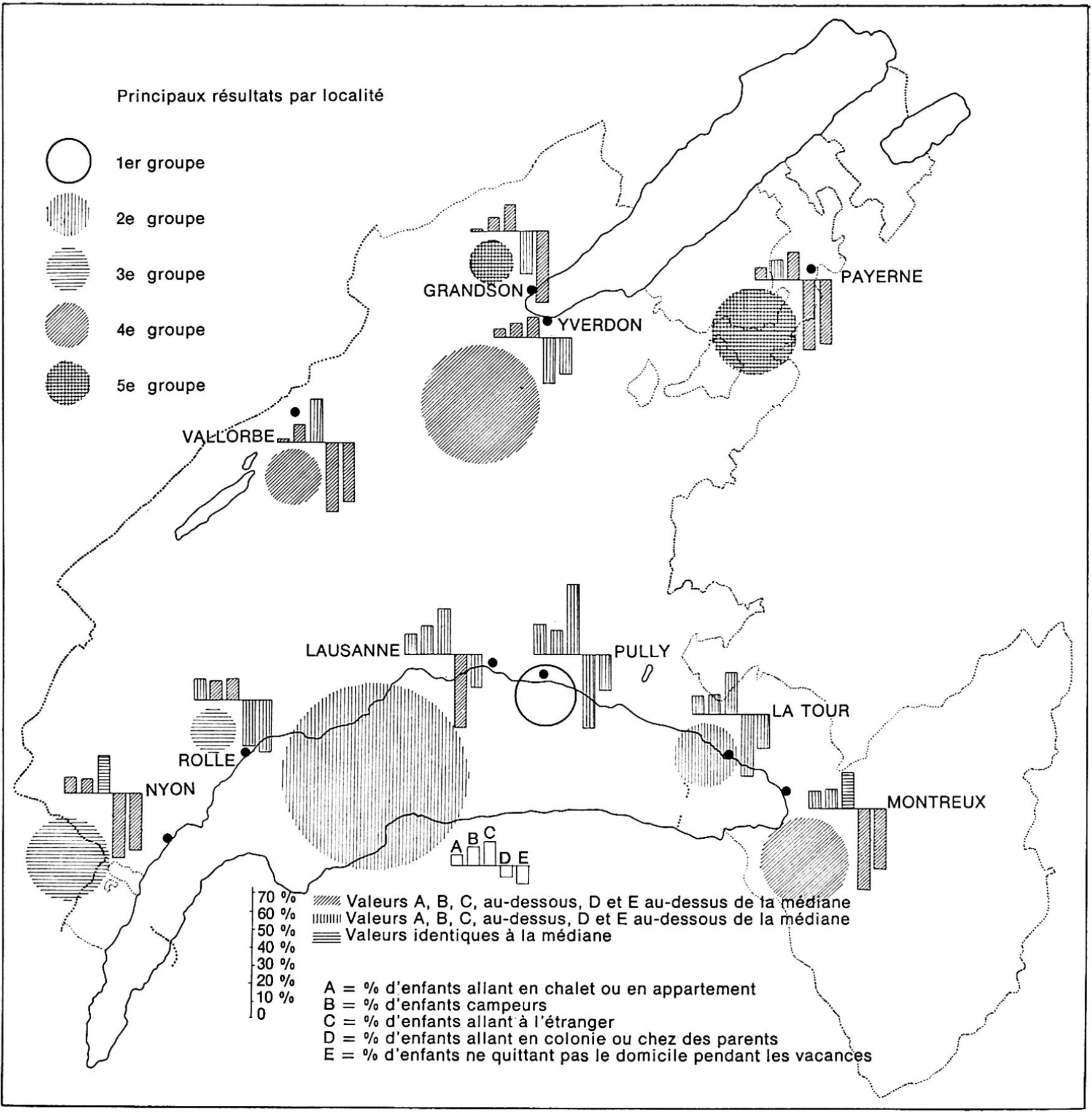

En conclusion, le genre de vacances des écoliers des classes primaires est lié à la catégorie socio-professionnelle dominante qui est elle-même étroitement en relation avec le mode de vie social. Les enfants des villes à forte population ouvrière sont moins mobiles dans leurs vacances que ceux des cités à prédominance tertiaire.

\section{Etude des résultats à l'intérieur de la commune de Lausanne}

Par chance, les résultats par classe sont disponibles pour l'ensemble de la ville de Lausanne, ce qui permet de pousser notre investigation dans deux direc- tions supplémentaires, tout d'abord par quartier, ensuite par catégorie d'âge.

\section{a) Etude des quartiers}

Les écoles primaires, à part certaines classes spéciales, sont encore largement des établissements de quartier. Il est donc posible de considérer que les élèves habitent dans un cercle de rayon assez restreint autour de l'école. Grâce à ce fait, un certain nombre d'éléments ont pu être mis en lumière.

Pour éviter de trop petits sous-groupes, nous n'avons retenu que les établissements d'une certaine importance (groupant au total 4745 élèves sur 5331). Les résultats sont indiqués au tableau 10. 


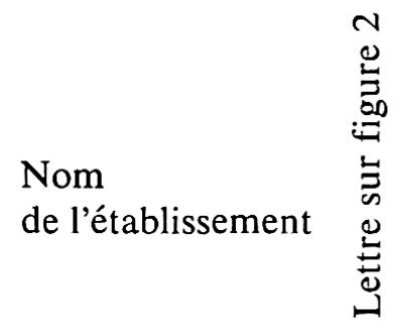

Questions

St-Roch

Villamont

Beaulieu

Borde

Prélaz

Croix-d'Ouchy

Floréal

Montriond

Croix-Rouges

Bellevaux

Malley

Chailly

La Sallaz

Pontaise

Montoie
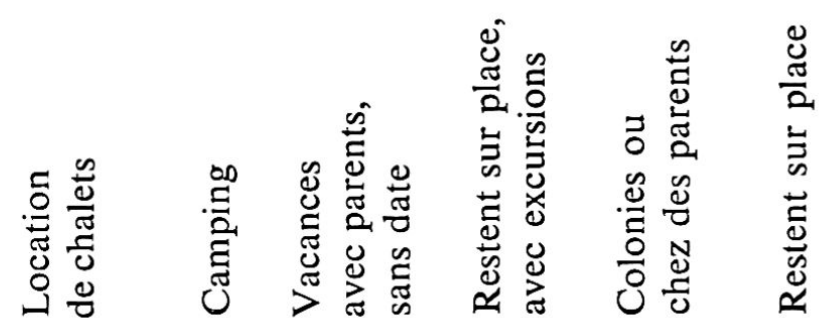

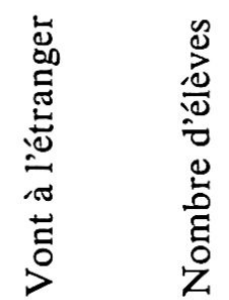

6

7

11

41

$14 \quad 37$

$11 \quad 37$

$14 \quad 37$

1240

$12 \quad 37$

$7 \quad 53$

$12 \quad 37$

$13 \quad 40$

$12 \quad 45$

$12 \quad 57$

$6 \quad 34$

$11 \quad 45$

$10 \quad 47$

$12 \quad 54$

$\begin{array}{lll}12 & 12 & 17\end{array}$

Total des élèves

Valeurs :

extrêmes

minimum 6

11

13

$\begin{array}{llll}\operatorname{maximum} & 19 & 20 & 32\end{array}$

$\begin{array}{rrr}6 & 34 & 2 \\ \mathbf{1 4} & \mathbf{5 7} & \mathbf{9}\end{array}$

15

32
En comparant les tableaux 3 et 10 , on constate que les variations au sein de la ville de Lausanne sont d'un autre ordre que les variations entre localités. A certains égards, les différences sont plus nettes, à d'autres, il y a une plus grande homogénéité. Pour les chalets, les résultats, en ville, sont plus groupés, de même que pour le camping. Dans ce dernier cas, les valeurs extrêmes sont toutes deux supérieures aux valeurs correspondantes du tableau 3: le camping est plus généralisé dans la ville qu'il ne l'est à travers le canton. Pour la question suivante, il y a peu de différence, alors qu'elles sont au contraire frappantes aux questions 6 et 8: il y a beaucoup moins d'enfants lausannois qui ne quittent pas du tout leur domicile pendant les vacances que d'enfants vaudois dans ce cas. Pour les questions 8 et 9 , l'amplitude entre quartiers est très faible. Quant à la question concernant ceux qui vont en colonies ou chez des parents, l'intervalle est presque le même mais les chiffres sont nettement plus élevés à Lausanne, justement du fait qu'il est plus rare que l'enfant de la capitale y reste tout l'été.

Par souci de simplification, nous avons tenté de grouper les principaux résultats en trois catégories: indices d'un quartier aisé, moyen, ouvrier. Les divers critères sont regroupés au tableau 11.

\section{Tableau 11}

Critères de classification des quartiers

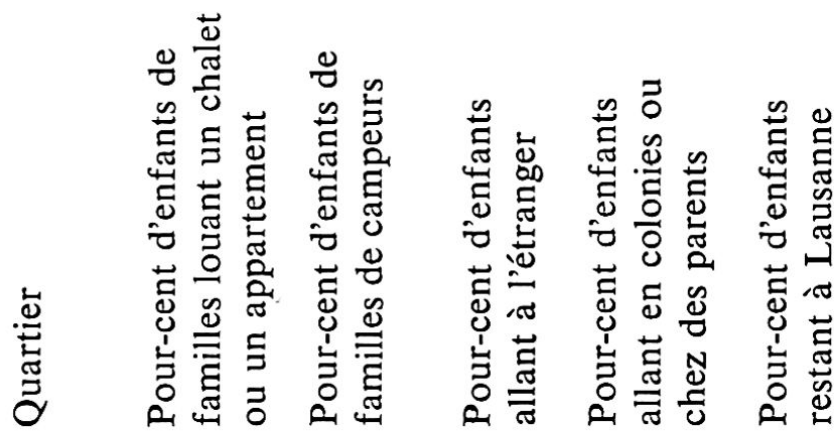

Questions $1-3 \quad 4 \quad 9 \quad 6 \quad 7 \quad 6$

aisé $\quad 16-19 \quad 17-20 \quad 29-32 \quad 34-37 \quad 12-15$

moyen 11-13 14-16 25-28 40-42 17-20

ouvrier $\quad 6-10 \quad 11-13 \quad 15-24 \quad 44-57 \quad 21-23$ 


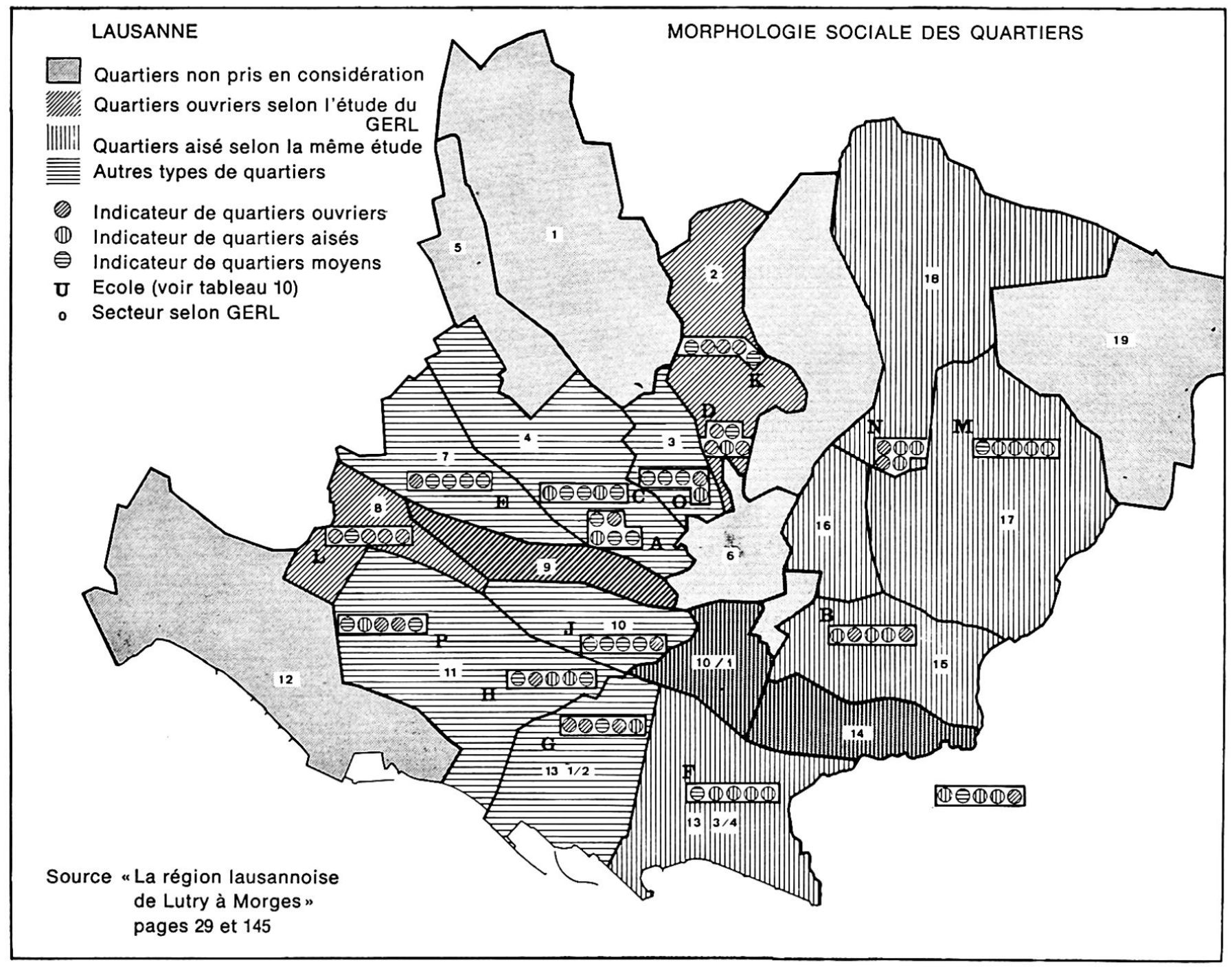

La question du camping n'est qu'un indicateur médiocre, en revanche les autres sont assez bons. Il faut par ailleurs remarquer que, à l'échelle européenne, Lausanne est, dans son ensemble, une ville tertiaire et que les quartiers ouvriers sont rares. D'autre part, nombreuses sont les parties de la ville où le mélange des catégories socio-professionnelles est assez poussé.

Cependant, la carte 2 est révélatrice d'une certaine morphologie sociale. Elle reprend le découpage en quartier mis au point par le groupe d'études de la région lausannoise 8 et tente de faire la synthèse des indications de notre enquête d'une part et des résultats du recensement fédéral de 1960 par branche d'activité 9 . Les traits essentiels ressortent très bien:

$1^{\circ}$ Un grand ensemble de quartiers aisés, à l'est, englobant aussi Pully, dont la limite coïncide à peu près avec celle de la grande cassure de la molasse.

$2^{\circ}$ Deux quartiers encore nettement ouvriers, l'un au nord, la Borde - Av. A.-Fauquex et rues avoisinantes 2, et l'autre à l'ouest, Malley 8, jouxtant la banlieue industrielle (Renens et Prilly). Le cas du quartier 13 (Croix-d'Ouchy - Place de Milan) est un peu particulier. Ce n'est vraisemblablement pas tant un secteur ouvrier qu'un endroit où subsistent un nombre important de logements anciens abritant des personnes de condition modeste.

$3^{\circ}$ Le reste de la ville est trop mélangé pour qu'il soit possible de le classer de manière bien tranchée.

\section{b) Comparaisons par âge}

Il est évident que la manière de passer ses vacances, pour un enfant ou un adolescent, varie avec l'âge. C'est pourquoi, il paraît utile de voir les résultats sous une autre forme, ce qui permettra aussi de nuancer les conclusions auxquelles nous avons abouti. 
Tableau 12

Résultats par degré (en pour-cent)

\begin{tabular}{|c|c|c|c|c|c|c|}
\hline & & & & & ants & \\
\hline 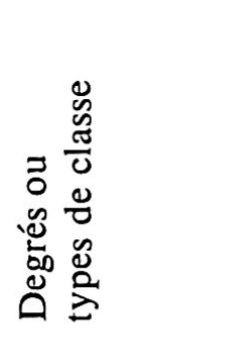 & 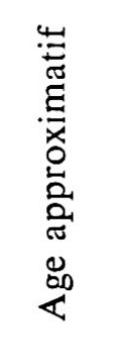 & 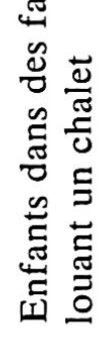 & 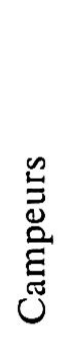 & 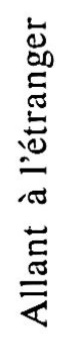 & 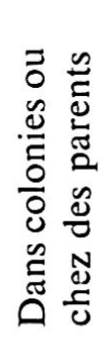 & 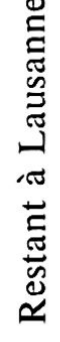 \\
\hline 3 & $9-10$ & 13 & 14 & 28 & 43 & 17 \\
\hline 4 & $10-11$ & 14 & 12 & 23 & 40 & 14 \\
\hline 5 & $11-12$ & 12 & 13 & 22 & 45 & 19 \\
\hline 6 & $12-13$ & 11 & 16 & 26 & 44 & 19 \\
\hline 7 & $13-14$ & 9 & 17 & 30 & 44 & 21 \\
\hline 8 & $14-15$ & 12 & 19 & 26 & 36 & 25 \\
\hline 9 & $15-16$ & 10 & 20 & 29 & 29 & 22 \\
\hline classe spécia & & 3 & 12 & 15 & 53 & 29 \\
\hline prim. sup. 6 & $12-13$ & 19 & 21 & 27 & 41 & 14 \\
\hline prim. sup. 7 & $13-14$ & 13 & 23 & 38 & 43 & 14 \\
\hline prim. sup. 8 & $14-15$ & 11 & 19 & 30 & 25 & 11 \\
\hline prim. sup. 9 & $15-16$ & 10 & 25 & 45 & 40 & 17 \\
\hline classe ménag & & 9 & 14 & 29 & 42 & 26 \\
\hline orientation $\mathrm{p}$ & & 9 & 10 & 23 & 28 & 37 \\
\hline
\end{tabular}

Ce tableau montre que l'âge ne joue pas toujours nettement un rôle, cependant son influence se retrouve dans les points suivants:

$1^{\circ}$ La part des campeurs augmente avec l'âge, mais aussi, nettement, avec le genre d'études. Les primaires supérieurs, peut-être plus curieux que les autres, recourent plus volontiers à ce mode de vacances.

$2^{\circ}$ Il en va de même pour les séjours à l'étranger où les pour-cent oscillent de 26 à $30 \%$ chez les primaires de 12 à 16 ans, alors qu'ils sont de $27 \grave{a}$ $45 \%$ chez les primaires supérieurs de même âge.

$3^{\circ}$ Pour les colonies de vacances et séjours chez des parents, le pourcentage est nettement plus faible à partir de 14 ans, ce qui doit être principalement lié au désir plus grand d'indépendance de l'adolescent ou aux limites fixées par les organisateurs.

$4^{\circ}$ La part de ceux qui restent à domicile croît avec l'âge, soit que l'adolescent devienne plus utile à ses parents, soit qu'il puisse se faire un peu d'argent sur place. C'est moins vrai pour les primaires supérieurs qui préfèrent le camping ou le voyage à l'étranger.

\section{Conclusion}

Nous avons progressivement vu comment diverses facettes de la réalité apparaissaient:

- Tout d'abord un degré de mobilité, d'intérêt et de moyens à disposition pour les vacances variant d'un lieu à l'autre du canton, opposant particulièrement les localités tertiaires aux villes industrielles.

- Certains quartiers de Lausanne, ensuite, s'individualisant, face à d'autres et soulignant les trois grands secteurs de la morphologie sociale de la ville.

- Enfin, les nuances plus fines dues à l'âge ou degré culturel des élèves, qui incitent les uns aux voyages et les autres aux activités sur place.

Ces résultats seront d'une part utiles à ceux qui cherchent à connaître les habitudes et les tendances des vacanciers suisses et d'autre part d'un intérêt non négligeable pour ceux qui analysent les localités du canton de Vaud.

\section{Remarques}

1 Voir le questionnaire en annexe

2 Orientation professionnelle

3 Non inclus dans les résultats cités

${ }^{4}$ H.-P. Schmidhauser, Cl. Kaspar: «Enquête d'opinion auprès des touristes d'été et d'hiver 1965/66 dans le canton de Vaud», Lausanne 1967, multicopié, Office cantonal vaudois de l'urbanisme.

5 Cités par M. J. Krippendorf dans un exposé donné à Nyon le 16 novembre 1967: «Facteurs de développement touristique - réflexions d'avenir"; selon l'enquête "Aisance et privations", réalisée en 1967, $58 \%$ des salariés de Suisse romande passent leurs vacances hors de chez eux.

6 Selon le "Répertoire des institutions sociales du canton de Vaud", Yverdon 1965

7 Chiffres exacts

8 GERL: "La région lausannoise de Lutry à Morges", Cahiers de l'aménagement régional 2, Lausanne 1966, p. 145.

9 Opuscule cité, p. 29. 